\title{
Terapia a laser de baixa potência no tratamento de lesões periféricas do nervo trigêmio em Odontologia: revisão de literatura
}

Low level laser therapy in the treatment of peripheral injuries of trigeminal nerve in Dentistry: literature review Terapia por láser de bajo nivel en el tratamiento de las lesiones periféricas del nervio trigémino en Odontología: revisión de la literatura

Ana Vitórya Alves de SOUZA

Lívia Fernandes PROBST ${ }^{2}$

Ellen Cristina GAETTI JARDIM ${ }^{2}$

Julio Cesar Leite da SILVA ${ }^{2}$

Graduanda de Odontologia (FAODO) da Universidade Federal de Mato Grosso do Sul (UFMS), 79070-900 Campo Grande - MS, Brasil ${ }^{2}$ Docente da Faculdade de Odontologia (FAODO) da UFMS, 79070-900 Campo Grande - MS, Brasil

\begin{abstract}
Resumo
A parestesia é um distúrbio neurossensorial decorrente de lesão nervosa que ocorre após uma intervenção odontológica e leva à perda de sensibilidade prolongada de uma região específica da face, além de outros sintomas, podendo ser resolvida espontaneamente após alguns dias ou meses, mas dependendo da extensão e tipo de lesão nervosa, este dano pode ser permanente. Com o avanço da tecnologia, os lasers vêm se popularizando na Odontologia, juntamente com os avanços em pesquisas. A terapia de fotobiomodulação com laser de baixa potência tem se mostrado como uma opção favorável ao tratamento de distúrbios neurossensoriais e aceleração do processo de reparo tecidual. Foi realizada uma revisão da literatura, a partir de buscas nas bases de dados do PubMed/MEDLINE, Biblioteca Virtual em Saúde (BVS), The Cochrane Library e Scielo, entre os anos de 2010 a 2020. O presente estudo tem como objetivo analisar a eficácia, a utilização terapêutica, e os mecanismos de ação da terapia a laser de baixa potência (LLLT), como também causas e fatores de risco relacionados aos procedimentos mais comuns de injúria nervosa. As recentes pesquisas envolvendo o uso da terapia de fotobiomodulação evidenciam a capacidade do laser em promover aceleração do processo de reparo tecidual, com fenômenos analgésicos, antiinflamatórios, cicatrizantes e regenerativos. Embora tenha havido grandes descobertas nos últimos anos, a literatura ainda carece de protocolos e ensaios clínicos sobre o uso do laser, verificando e comprovando seus efeitos sobre lesões nervosas, devendo este ser o foco de pesquisas futuras.
\end{abstract}

Descritores: Terapia com Luz de Baixa Intensidade; Parestesia; Fotobiomodulação.

\section{Abstract}

Paresthesia is a neurosensory disorder resulting from nerve damage that occurs after a dental intervention and leads to prolonged loss of sensation in a specific region of the face, in addition to other symptoms, which can be resolved spontaneously after a few days or months, but depending on the extension and type of nerve damage, this damage can be permanent. With the advancement of technology, lasers have become popular in dentistry, along with advances in research. The photobiomodulation therapy with low level laser has been shown to be a favorable option for the treatment of neurosensory disorders and acceleration of the tissue repair process. A literature review was carried out, based on searches in the databases of PubMed / MEDLINE, Virtual Health Library (VHL), The Cochrane Library and Scielo, between 2010 and 2020. The present study aims to analyze the effect, therapeutic use, and the mechanisms of action of low level laser therapy (LLLT), as well as causes and factors related to the most common nerve injury procedures. Recent research involving the use of photobiomodulation therapy shows the laser's ability to accelerate the tissue repair process, with analgesic, anti-inflammatory, healing and regenerative phenomena. Although there have been major discoveries in the years, the literature still lacks protocols and clinical trials on the use of lasers, verifying and proving their effects on nerve injuries, which should be the focus of future research.

Descriptors: Low-Level Light Therapy; Paresthesia; Photobiomodulation.

\section{Resumen}

La parestesia es un trastorno neurosensorial resultante del daño de los nervios que se produce tras una intervención dental y conduce a una pérdida prolongada de la sensibilidad en una región específica del rostro, además de otros síntomas, que pueden resolverse espontáneamente a los pocos días o meses, pero que se prolongan en extensión y tipo de daño nervioso, este daño puede ser permanente. Con el avance de la tecnología, los láseres aprenden a hacerse populares en odontología, junto con los avances en la investigación. Una terapia de fotobiomodulación con láser de baja potencia tiene que ser una opción favorable para el tratamiento de los trastornos neurosensoriales y la aceleración del proceso de reparación tisular. Se realizó una revisión bibliográfica, a partir de búsquedas en las bases de datos de PubMed / MEDLINE, Virtual Health Library (BVS), The Cochrane Library y Scielo, entre 2010 y 2020. El presente estudio tiene como objetivo analizar la efecto, uso terapéutico y los mecanismos de acción de la terapia con láser de baja potencia (LLLT), así como las causas y factores relacionados con los procedimientos de lesión nerviosa más comunes. Investigaciones recientes que involucran el uso de terapia de fotobiomodulación muestran la capacidad del láser para acelerar el proceso de reparación de tejidos, con fenómenos analgésicos, antiinflamatorios, cicatrizantes y regenerativos. Aunque ha habido grandes descubrimientos a lo largo de los años, la literatura aún carece de protocolos y ensayos clínicos sobre el uso de láseres, verificando y demostrando sus efectos sobre las lesiones nerviosas, que deberían ser el foco de futuras investigaciones.

Descriptores: Terapia de Luz de Bajo Nivel; Parestesia; Fotobiomodulación.

INTRODUÇÃO

A parestesia oral é uma complicação odontológica temporária ou permanente, associada a exodontias de terceiros molares mandibulares, inserção de implantes, cirurgias ortognáticas e outros procedimentos cirúrgicos ou não-cirúrgicos ${ }^{1}$, que podem levar à perda de sensibilidade prolongada nas áreas inervadas pelo feixe lesado, tendo suas causas ainda hoje muito discutidas. Com isso, sabe-se que o retorno da normalidade depende da regeneração das fibras nervosas lesadas ou da remissão das causas secundárias que estão gerando a parestesia, como a reabsorção do sangramento local, e a redução do edema e da inflamação, que podem estar comprimindo o nervo e gerando a parestesia ${ }^{2}$. 
Os principais sintomas decorrentes desta complicação incluem a perda de sensibilidade em uma região específica e localizada, sensações de choque elétrico, dormência, queimação ou formigamento ${ }^{3}$. As injúrias nervosas periféricas do nervo trigêmio, (quinto par de nervos cranianos com ramos oftálmico, maxilar e mandibular) são uma das complicações pós-operatórias mais sérias decorrentes de procedimentos cirúrgicos odontológicos, e podem resultar em distúrbios neurossensoriais como a parestesia (sensação anormal de sensibilidade), hipoestesia (sensibilidade reduzida após estímulo), hiperalgesia (aumento da dor pelo estímulo que normalmente provoca dor) ou, ainda pior, alguma forma de disestesia (sensação anormal desagradável estimulada ou não) como alodinia (dor devido a um estímulo que geralmente não provoca a dor) ou até mesmo a completa perda de sensibilidade completa (anestesia) ${ }^{4}$.

Todas estas alterações neurossensoriais podem afetar seriamente a capacidade do paciente de realizar atividades diárias, como beber e comer, e também pode levar a lesões traumáticas dos tecidos moles, como lábios ou bochechas, durante a mastigação ${ }^{5}$. Isso pode causar muita apreensão e ansiedade ao paciente, sendo importante que o cirurgiãodentista saiba como tratar e prevenir essa parestesia, pois em casos de iatrogenia, podese culminar a processos legais administrativos ou jurídicos.

A terapia a laser de baixo nível (low level laser therapy - LLLT) ou, mais recentemente chamada de terapia de fotobiomodulação, é uma forma de tratamento que utiliza fontes de luz não ionizantes no espectro visível e infravermelho. É um processo não térmico que envolve cromóforos endógenos que provocam eventos fotofísicos e fotoquímicos em várias escalas biológicas. Esse processo resulta em resultados terapêuticos benéficos, incluindo, entre outros, o alívio da dor ou inflamação, imunomodulação e promoção da cicatrização de feridas e regeneração de tecidos ${ }^{6}$.

Esta terapia vem sendo utilizada e pesquisada amplamente nos dias atuais, pois diante de um quadro de parestesia, sabe-se que a maioria dos casos se resolvem espontaneamente, mas alguns podem ser persistentes ${ }^{7}$, deixando o cirurgião-dentista com poucas opções terapêuticas, como a prescrição de medicamentos do complexo B (Etna, Citoneurin) e anti-inflamatórios, fisioterapia, ou, quando mais grave, o encaminhamento para neurocirurgiões.

Atualmente, muito se tem publicado sobre os efeitos do laser de baixa potência, principalmente de sua eficácia na resolução e diminuição do desconforto de lesões ulceradas intrabucais, como úlceras aftosas recorrentes e traumáticas ${ }^{8}$ e também no auxílio do tratamento de outras doenças orais como líquen plano, pênfigo vulgar, herpes simples recorrente e osteonecrose da mandíbula relacionada a bisfosfonatos, entre outros ${ }^{9}$. No entanto, faltam ainda comprovações suficientes que demonstrem sua efetividade no tratamento de lesões nervosas orais e distúrbios neurossensoriais decorrentes. Os lasers possuem efeitos biológicos benéficos, de caráter analgésico, anti-inflamatório e cicatrizante, por meio do fenômeno da bioestimulação ${ }^{10}$, no entanto, sabe-se que estes efeitos benéficos só são obtidos com a aplicação e indicação corretas. Para isso, é necessário que se estabeleçam protocolos de aplicação para cada tipo de intervenção, ou seja, devemos aplicar a radiação laser de acordo com o tipo de tecido alvo e de efeito desejado.

Assim, o presente artigo tem como objetivo realizar uma revisão da literatura, de modo a apresentar informações resumidas referentes ao uso clínico do laser de baixa potência na terapia de fotobiomodulação, para resolução da parestesia e outros distúrbios neurossensoriais decorrentes de trauma nervosos periféricos trigeminais após procedimentos odontológicos de rotina. Além de abordar as causas dos traumas nervosos, fatores de risco durante procedimentos como exodontia de terceiros molares, cirurgias ortognáticas, implantes e tratamentos endodônticos, eficácia clínica do laser em lesões nervosas periféricas trigeminais, as considerações anatômicas e histológicas das lesões nervosas periféricas e os mecanismos de ação do laser de baixa potência nos tecidos danificados, com suas ações moleculares e celulares.

MATERIAL E MÉTODO

Esta revisão de literatura foi realizada a partir de buscas nas bases de dados do PubMed/MEDLINE, Biblioteca Virtual em Saúde (BVS), The Cochrane Library e Scielo. Baseado no acrônimo PICO, os artigos foram pesquisados por termos extraídos do Mesh e Emtree para possibilitar estratégia de busca, sem a restrição de idioma. Os artigos originais foram selecionados de acordo com ano de publicação (2010 a 2020) e relevância sobre o assunto. A estratégia de pesquisa envolveu o uso dos seguintes termos: "low level laser therapy", "low level light therapy", 
"photobiomodulation therapy", "neurosensory disturbance", "nerve injury", "paresthesia", "alveolar inferior nerve injury", "risk factors", "dentistry".

Também foram conduzidas buscas manuais adicionais por outros artigos não recuperados nas buscas, a partir da análise das referências dos artigos relevantes incluídos para leitura do texto completo. Os artigos foram selecionados com base nos critérios de elegibilidade pré-estabelecidos. Como critérios de inclusão foram considerados os artigos publicados entre 2010 a 2020, nas categorias estudos de revisão sistemática e meta-análises, ensaios clínicos randomizados, revisão de literatura e casos clínicos. Os critérios de exclusão consideraram os artigos publicados antes de 2010, os artigos sobre uso do LLLT em nervos motores e os estudos 'in vitro'.

A partir da seleção dos artigos pelo título relacionado ao tema principal, foi realizada a análise dos resumos de cada artigo para confirmar sua relação ao assunto, abordando o uso do laser de baixa potência em lesões nervosas periféricas trigeminais. Depois de ter excluído os artigos não relevantes pelo resumo, foi feita a leitura completa dos textos para análise e citação no presente estudo.

REVISÃO DA LITERATURA

\section{- Causas das parestesias}

A injúria nervosa e consequente disfunção neurossensorial, em odontologia, pode se desenvolver após vários tipos de procedimentos, mas a maioria das parestesias orais é causada por trauma direto associado ao procedimento cirúrgico. No entanto, estudos demonstraram que a parestesia também pode ocorrer devido à ação tóxica dos anestésicos locais, principalmente em bloqueios com o uso da Articaína e Prilocaína, em concentrações a $4 \%{ }^{11}$

A remoção de terceiros molares impactados é um dos procedimentos cirúrgicos mais comuns na área dentofacial, geralmente associado a efeitos colaterais, como dor, inchaço e trismo nos primeiros dias após a operação ${ }^{12}$.

A lesão do nervo alveolar inferior (NAI) é uma das as complicações mais graves após a extração do terceiro molar inferior (3M), uma vez que a incidência de lesão pós-operatória do NAl é maior quando as raízes do dente estão em íntimo contato com a cortical óssea do canal alveolar inferior, ou seja, a relação do ápice dentário com o canal mandibular que indicará o risco de danos à $\mathrm{IAN}^{13}$.

Em relação às cirurgias ortognáticas, a realização da osteotomia sagital bilateral e osteotomia horizontal deslizante da sínfise mandibular apresentam risco de distúrbios neurossensoriais devido à proximidade dos locais da osteotomia com o nervo alveolar inferior e nervo mental, respectivamente ${ }^{14}$.

Devido à isso, a lesão do nervo alveolar inferior é uma das complicações mais comuns após a osteotomia sagital bilateral, pois a manipulação do nervo durante a dissecção e separação do ramo, o estiramento excessivo do nervo, e a compressão do nervo durante a fixação tornam o procedimento muito arriscado a lesões nervosas. A parestesia também pode ocorrer devido a lesão direta por serra, broca e cinzel utilizados para a osteotomia ou devido a lesão por hematoma ou edema no canal nervoso ${ }^{15}$.

Outro tipo de procedimento cirúrgico que está relacionado com a parestesia é a colocação de implantes, podendo ocorrer durante as injeções de anestésico local, execução da osteotomia ou durante 0 ato de colocação do implante, principalmente em intervenções na mandíbula. $\mathrm{O}$ calor produzido pela broca durante a perfuração para colocação implante pode passar ao tecido neural circundante, como ao NAl, e causar lesão nervosa, pois estes tecidos são muito sensíveis e facilmente danificados por estímulos térmicos ${ }^{5}$.

Além disso, o contato entre a broca de implante e a parede óssea pode ocorrer durante o procedimento de osteotomia e escorregar devido à presença de osso menos compacto na direção da perfuração; esse escorregamento pode causar trauma mecânico ao nervo ${ }^{5}$.

Outras causas não cirúrgicas associadas à parestesia e outras disfunções neurossensoriais também foram relatadas literatura. São os casos de tratamentos endodônticos com preparo e alargamento excessivo do canal radicular que resulta em rompimento da constrição apical, que por sua vez é responsável pela extrusão de irrigantes, medicamentos e materiais de obturação (gutapercha e cimentos) na região periapical e subsequente lesão química e mecânica dos nervos $^{16,17}$.

A movimentação ortodôntica também pode, embora muito raramente, provocar distúrbios neurossensoriais secundários. Em certas situações clínicas, as raízes dos dentes posteriores podem estar em proximidade com 0 canal do NAI, elevando o risco de o movimento de torque gerar compressão ao nervo, e causar parestesia temporária ${ }^{18}$.

- Considerações anatômicas e histológicas das lesões nervosas 
As células não neuronais e os tecidos conjuntivos que circundam os axônios neuronais fornecem um suporte complexo para o nervo e são importantes na compreensão e classificação das lesões nervosas. A camada que envolve os axônios individuais é a camada estrutural mais profunda, o endoneuro. Ao redor do endoneuro, o perineuro agrupa axônios para formar fascículos; e a camada mais externa de tecido conjuntivo do nervo é 0 epineuro ${ }^{19}$ (Figura 1). Este número de camadas varia entre os tipos de fibras nervosas, e explica a maior suscetibilidade de o nervo lingual sofrer um trauma do que o nervo alveolar inferior após bloqueios anestésicos nessa área, devido à sua tendência a ser uni-fascicular e seu menor espessamento do perineuro. Nervos multifasciculares têm maior tecido epineural, e isso pode permitir que o edema ou hemorragia induzida por trauma sejam dispersos com menos pressão nos axônios, causando menos danos $^{20}$.

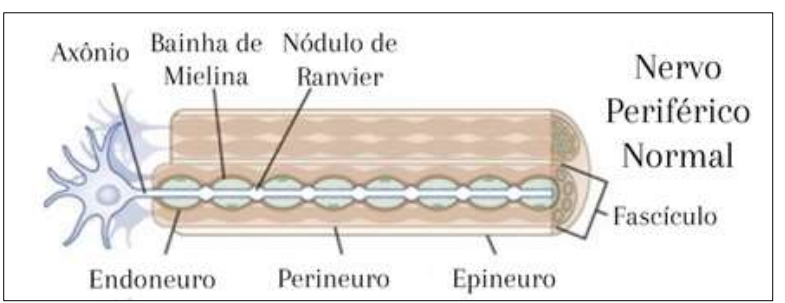

Figura 1: Anatomia do nervo periférico ${ }^{19}$. (Tsao B, Boulis N, Bethoux F, Murray B. Trauma of the Nervous System, Peripheral Nerve Trauma. In: Daroff: Bradley's Neurology in Clinical Practice, 6th ed. 2012 p 984-1001.Cortesia de Cleveland Clinic, 2006. Illustrator, David Schumick, BS, CMI) Modificado por SOUZA, AVA.

Quanto às classificações das lesões aos nervos periféricos, Seddon ${ }^{21}$, em 1943, foi o primeiro a classificar as lesões nervosas em três categorias, com base na presença de desmielinização e na extensão dos danos aos axônios e aos tecidos conjuntivos do nervo, sendo ainda hoje a classificação mais utilizada. A forma mais branda de lesão é chamada neurapraxia, definida por desmielinização focal sem danificar os axônios, ocorrendo tipicamente por leve compressão ou tração do nervo e resulta em uma diminuição na velocidade de condução, dependendo da gravidade da desmielinização. A qualidade da recuperação é excelente, geralmente espontânea. O próximo nível é chamado axonotmese, que envolve dano direto aos axônios, com completa interrupção dos axônios, além da desmielinização focal, mas com preservação das estruturas de suporte, mantendo a continuidade dos tecidos conjuntivos do nervo. A recuperação tende a ser espontânea e de boa qualidade, porque as fibras regeneradoras são guiadas em seus caminhos adequados por suas bainhas intactas. Já a forma mais grave de lesão é chamada neurotmese, que é uma transecção completa dos axônios e camadas do tecido conjuntivo, em que é observada a descontinuidade completa do nervo, ou seja, todas as partes essenciais do nervo são destruídas (Figura 2).

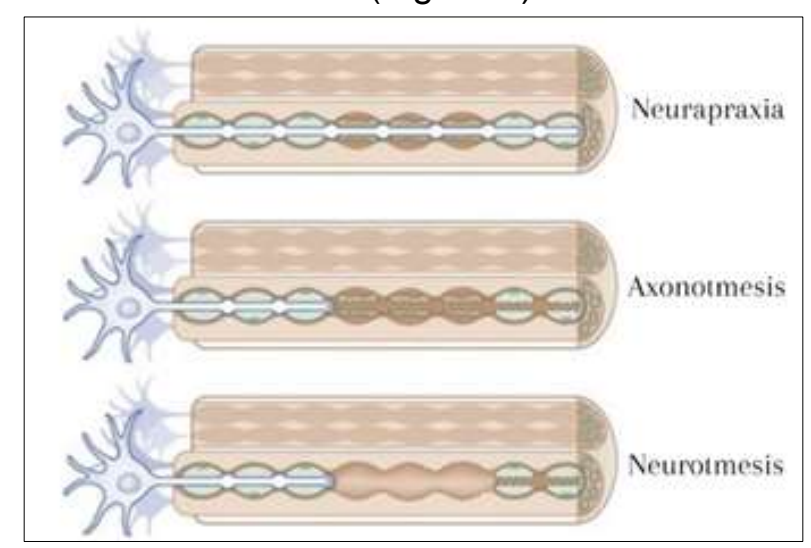

Figura 2: Classificação das lesões nervosas periféricas de Seddon ${ }^{21}$. (Tsao B, Boulis N, Bethoux F, Murray B. Trauma of the Nervous System, Peripheral Nerve Trauma. In: Daroff: Bradley's Neurology in Clinical Practice, 6th ed. 2012 p 984-1001.- Cortesia de Cleveland Clinic, 2006. Illustrator, David Schumick, BS, CMI.) Modificado por SOUZA, AVA.

Sunderland, posteriormente, expandiu e aperfeiçoou essa classificação para distinguir a extensão dos danos nos tecidos conjuntivos, considerando algumas limitações de Seddon ${ }^{22}$. Em seu esquema de classificação, os graus I e $\mathrm{V}$ correspondiam à neurapraxia e neurotmese de Seddon, respectivamente. No entanto, Grau II-IV são todas as formas de axonotmese com quantidades crescentes de danos no tecido conjuntivo. No Grau II, observa-se dano no axônio, acompanhada pela desintegração de sua bainha de mielina, sem danos ao tecido conjuntivo circundante; no grau III envolve danos ao endoneuro, e no grau IV inclui danos ao perineuro ${ }^{19}$ (Tabela 1 ).

Tabela 1. Classificação de lesões nervosas de Seddon e Sunderland (Menorca RMG, Fussell TS, Elfar JC. Peripheral Nerve Trauma: Mechanisms of injury and recovery. Hand Clinics. 2013. p. 317-30)

\begin{tabular}{l|l|l|}
\hline Seddon & Sunderland & Dano \\
\hline
\end{tabular}

\begin{tabular}{l|l|l|}
\hline Seddon & Sunderland & Dano \\
\hline Axounotmesia & Grau I & Desmielinização focal de um segmento \\
\hline Grau II & Axônio danificado com endoneuro intacto \\
\hline
\end{tabular} $\begin{array}{lll}\text { Axâ III } & \text { Axônio e endoneuro danificados e perineuro intacto }\end{array}$ \begin{tabular}{l|l|l}
\hline Axonotmesis & Grau IV & Axônio, endoneuro, perineuro danificados, com
\end{tabular} \begin{tabular}{l|l|l} 
& & epineuro intacto \\
\hline Neurotmesis & Grau V & Transecção completa do nervo
\end{tabular}

- Fatores de risco às lesões nervosas

A exodontia do terceiro molar inferior é um dos procedimentos mais comuns na prática cirúrgica oral, e, devido a isso, o comprometimento neurossensorial é uma complicação comum devido à relação de proximidade entre as raízes do dente e o canal mandibular. O conhecimento de fatores de risco pode ajudar a prever lesões nervosas, além orientar as recomendações no manejo de pacientes com alto risco de lesão ao NAI após a remoção dos terceiros molares ${ }^{23}$.

Os principais fatores associados à dificuldade de extração de terceiros molares em casos difíceis ou complexos são: o aumento da 
idade do paciente e excesso de peso, cirurgiões com pouca experiência, uso de técnicas cirúrgicas complexas e fatores radiológicos adversos como profunda impactação, angulação desfavorável e morfologia radicular com estreita relação com o segundo molar, seio maxilar ou canal mandibular ${ }^{24}$.

Para isso, a radiografia panorâmica (RP) é o exame de imagem que a maioria dos cirurgiões-dentistas usa para observar a localização do terceiro molar inferior, grau de impacção, e avaliar o risco de danos ao nervo alveolar inferior ${ }^{25}$. Segundo Su et al. ${ }^{26}$, existem alguns sinais vistos na radiografia panorâmica que podem predizer riscos de lesão ao NAI, que incluem o desvio do canal, a interrupção da linha branca do canal (cortical óssea) e escurecimento da raiz, sendo que estes sinais podem ser considerados suficiente para julgar 0 risco de lesão pós-cirúrgica do 3M (Figura 3).

Com base nisso, nem sempre se pode prever com precisão a presença ou ausência dos riscos de dano ao NAl com as radiografias panorâmicas, por causa de suas limitações de imagem, sendo necessário, muitas vezes, indicar exames de diagnóstico mais detalhados e aprofundados. Araujo et al. $^{27}$ mostraram que a TCCB, com imagens nos planos coronal, sagital e axial, contribui para uma avaliação ótima dos riscos e, como consequência, para um planejamento cirúrgico mais adequado, comparado à radiografia panorâmica, embora não altere a abordagem cirúrgica quando comparada à radiografia panorâmica; no entanto, a tomografia CB é considerada um método de imagem útil para entender a relação entre os terceiros molares inferiores e canal mandibular.

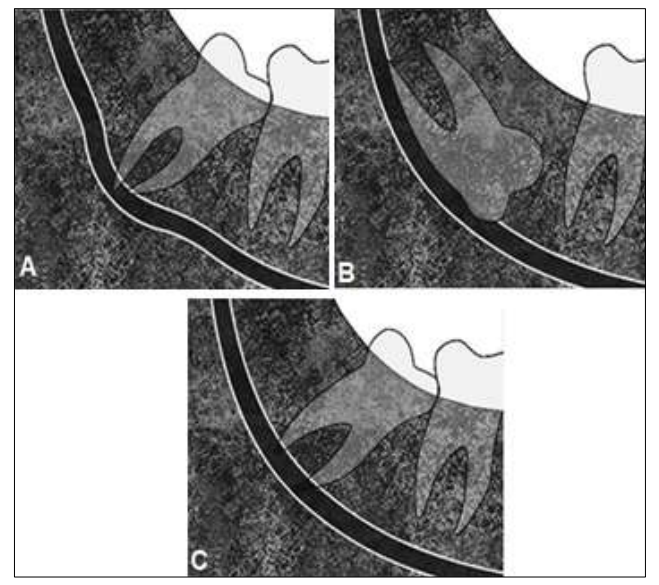

Figura 3: Sinais vistos na radiografia panorâmica que podem predizer riscos de lesão ao NAI na exodontia 3M: (A) o desvio do canal, (B) a interrupção da linha branca do canal (cortical óssea) e (C) escurecimento da raiz. Modificado por SOUZA, AVA. (Kang F, Sah MK, Fei G. Determining the risk relationship associated with inferior alveolar nerve injury following removal of mandibular third molar teeth: A systematic review. J Stomatol Oral Maxillofac Surg. Elsevier Masson SAS; 2020. p. 63-9).

Aliado à maior disponibilidade da tecnologia 3D atualmente, os profissionais passaram a indicar a tomografia CB mais frequentemente $\mathrm{e}$, eventualmente, de forma incorreta, expondo o paciente a uma dose excessiva de radiação. No entanto, não há fortes evidências de que a TCCB tem efeito na redução de distúrbios neurossensoriais após a remoção do $3 \mathrm{M}$ quando comparado às radiografias panorâmicas ${ }^{28}$.

Clé-Ovejero et al. ${ }^{29}$ mencionam que a TCCB é indicada quando sinais suspeitos de proximidade com o canal mandibular são revelados na radiografia panorâmica, sendo que a imagem 3D pode ser útil ao fornecer informações adicionais e detalhadas sobre a relação entre o terceiro molar inferior e o NAI, dando maior confiança ao cirurgião ao realizar o procedimento e, consequentemente, evitando movimentos prejudiciais durante a cirurgia que podem causar danos nervosos e distúrbios neurossensoriais pós-operatórios.

Em relação à cirurgia de implantes dentais, a sensação alterada persistente ou transitória pode indicar distúrbio neurossensorial associado a uma lesão do nervo alveolar inferior e ramo mentoniano, podendo também causar dor intensa debilitante nos pós-operatório.5 Em cirurgia de implante com procedimentos convencionais, o risco de alteração sensorial imediatamente após a cirurgia é substancial $(13 \%)$, sendo que na maioria dos casos, a resolução é espontânea, e dentro de 6 meses após a cirurgia, $80 \%$ dos pacientes retornaram à sensação normal e um ano após a cirurgia, 91\% dos casos tem resolução dos distúrbios neurossensoriais ${ }^{30}$.

A modalidade mais empregada para medir o osso vertical até o canal do $\mathrm{n}$. alveolar inferior é a radiografia panorâmica, no entanto, a recomendação de tomografias cone-beam pode ser útil sempre que informações mais detalhadas sejam necessárias, como nos casos onde o comprimento vertical medido é inadequado e a opção de inserir os implantes lateralmente ao canal alveolar inferior é considerada, servindo à equipe cirúrgica como um exame abrangente para alcançar os melhores resultados ${ }^{31}$.

Portanto, considerando os efeitos debilitantes resultantes da lesão ao NAI, todos os pacientes submetidos à reabilitação oral através de implantes dentários devem ser avaliados com imagens de tomografias computadorizadas de feixe cônico, que também irá auxiliar no planejamento do procedimento e na redução das complicações nervosas em implantodontia ${ }^{32}$.

A cirurgia ortognática é um procedimento realizado com a finalidade de correção das 
deformidades dentofaciais. Le Fort I e osteotomias sagitais bilaterais do ramo da mandíbula são as técnicas cirúrgicas mais comumente usadas, sendo o comprometimento neurossensorial a complicação mais comum no pós-operatório $(65,1 \%)^{33}$.

Durante a execução da osteotomia sagital bilateral, danos ao nervo alveolar inferior podem resultar de tração do nervo durante a remoção ou manipulação do fragmento ósseo distal, fixação incorreta do corte ósseo, e durante a dissecção óssea com a broca ou outros instrumentos cortantes ${ }^{34}$.

Com isso, a sensação alterada que pode se desenvolver após a cirurgia ortognática que envolva a osteotomia mandibular é uma complicação inevitável, sendo a lesão do nervo alveolar inferior uma das complicações mais comuns neste tipo de cirurgia, e na maioria dos casos, ocorre a resolução espontânea, mas alguns podem se tornar persistentes ${ }^{15}$.

Existem alguns fatores de risco que influenciam no tempo de recuperação dos distúrbios neurossensoriais decorrentes de cirurgias ortognáticas, sendo significativamente afetado pela idade, pois quando mais avançada pode aumentar o risco de dano permanente, a quantidade de movimento do fragmento da mandíbula $(>7 \mathrm{~mm})$ e a manipulação do nervo, que pode aumentar o tempo de recuperação ou tornar o dano permanente. Nesses casos, quanto mais invasiva a operação for, mais numerosas e relevantes são as possíveis complicações ${ }^{35}$.

Para isso, o paciente deve ser informado de possíveis riscos antecipadamente, por meio de termos de consentimento - com seu nível de cultura e capacidade de entender adequados, especialmente em situações de alto risco, quando um procedimento invasivo e difícil é esperado, em que pode haver uma diminuição temporária ou permanente da integridade física, como no caso de procedimentos cirúrgicos nos quais existe o risco de lesões nervosas. Assim, se o CD não avisar o paciente sobre todos os riscos relevantes, ele arrisca uma ação por negligência, pois o consentimento informado desempenha um papel essencial na minimização de litígio ${ }^{36}$.

- Mecanismos de ação do laser de baixa potência

O termo LASER é um acrônimo para 'Light Amplification by the Stimulated Emission of Radiation' (Amplificação de luz pela emissão estimulada de radiação). Esta tecnologia, introduzida na Odontologia na década de 60, vem sendo usada em várias especialidades, e pode ser classificado como gás, líquido, sólido e semicondutor, que especifica o tipo do raio laser que será emitido. Sua aplicabilidade varia em tecidos duros e moles, como na prevenção à cárie, hipersensibilidade dentinária, cicatrização de lesões herpéticas, na remoção de tecido hiperplásico e hemostasia, frenectomia e outros procedimentos $^{37}$.

A laserterapia de baixa potência (LLLT) ou fotobiomodulação, do inglês, photobiomodulation - PBM emprega a luz visível, geralmente vermelha, ou a luz infravermelha próxima gerada a partir de um sistema sólido, como o diodo emissor de luz (LED), para estimular a cura, aliviar a dor e reduzir a inflamação. (Figura 4) Os mecanismos moleculares e celulares da laserterapia de baixa potência sugerem que os fótons irradiados são absorvidos pelas mitocôndrias, estimulando a produção de ATP em alta quantidade e alteração dos níveis de espécies reativas de oxigênio (ERO), que ativam fatores de transcrição que induzem a transcrição de genes responsáveis pelos efeitos benéficos do $\operatorname{laser}^{38}$.

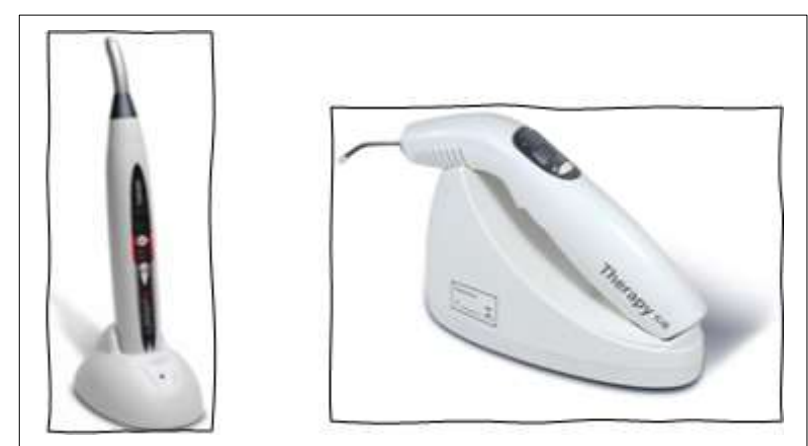

Figura 4: Lasers de Baixa Potência. (A) Aparelho Laser Duo - Recover MM Optics, semicondutor (GaAlAs e InGaAIP), com comprimentos de onda $660 \mathrm{~nm}$ (laser vermelho) e 808nm (laser infravermelho), $100 \mathrm{~mW}$; (B) Aparelho Laser DMC Therapy ILIB, Luz diodo, comprimento de onda 660 nm (vermelho), $100 \mathrm{~mW}$. (Fonte: manual de instruções MM Optics e DMC)

Hamblin M. et al. ${ }^{10}$ propõem que a densidade de potência insuficiente ou a aplicação do laser em tempo muito curto não terá efeito sobre a patologia, e que densidade e/ou tempo de potência em excesso podem ter efeitos inibitórios indesejados.

- Mecanismos moleculares

O citocromo C oxidase (CCO) é a unidade IV, enzima terminal, na cadeia de transporte de elétrons mitocondriais, sendo o responsável por absorver a luz irradiada, trasmitir fotossinais e induzir o aumento na síntese de ATP após receber o estímulo da luz. Segundo23, parece que a PBM aumenta a disponibilidade de elétrons para a redução do oxigênio molecular no centro catalítico do CCO, aumentando $\mathrm{o}$ potencial de membrana mitocondrial (PMM) e os níveis de ATP, AMPc, NO (óxido nítrico) e Cálcio, como resultado do influxo de $\mathrm{Ca} 2+$ do ambiente extracelular. (Figura 5) Isso explica o amplo uso da luz 
vermelha ou infravermelha na laserterapia, pois esses comprimentos de onda têm uma penetração muito melhor nos tecidos do que a luz azul ou verde, obtendo melhores respostas à radiação laser ${ }^{39}$.

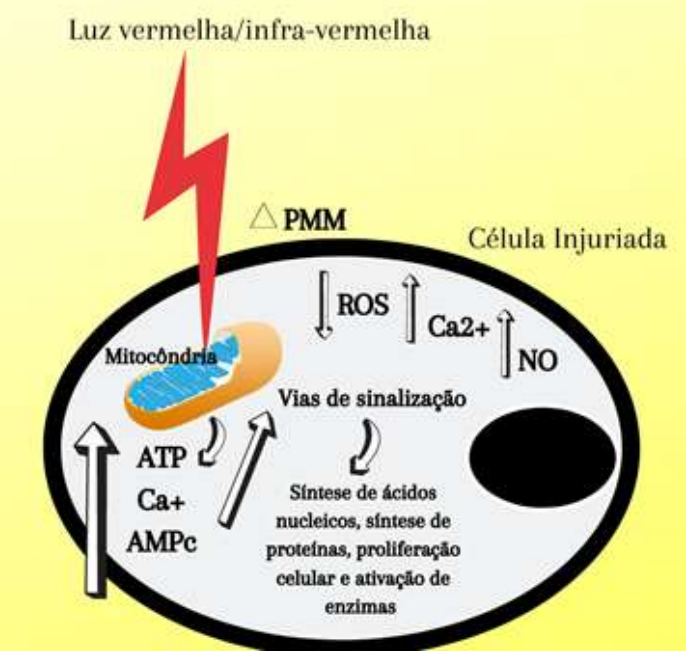

Figura 5: Mecanismos de ação da luz vermelha/infra-vermelha. Ao incidir sobre a célula injuriada, a luz vermelha/infra-vermelha altera o potencial de membrana mitocondrial, provocando o aumento dos níveis de ATP, Cálcio, AMPc, e NO, diminuição de ROS, e ativa vias de sinalização que induzem a síntese de DNA/RNA e proliferação celular. (Freitas LF, Hamblin MR. Proposed Mechanisms of Photobiomodulation or Low-Level Light Therapy. IEEE Journal on Selected Topics in Quantum Electronics. Institute of Electrical and Electronics Engineers Inc.; 2016 May 1;22(3).) Modificado por SOUZA, AVA.

A atividade do citocromo c oxidase é inibida pelo óxido nítrico (NO), mas a irradiação com laser pode reverter essa inibição fotodissociando o NO de seus locais de ligação, e aumentando a taxa de respiração. Além disso, a terapia de fotobiomodulação tem a capacidade de diminuir os níveis de ERO em células e tecidos injuriados, produzindo uma mudança no potencial de redução da célula, e isso induz a ativação de inúmeras vias de sinalização intracelular, tais como síntese de ácidos nucleicos, síntese de proteínas, ativação de enzimas e progressão do ciclo celular. A partir dessas vias, ocorre a ativação de fatores de transcrição responsáveis pela expressão gênica relacionada à proliferação de células de reparo tecidual ${ }^{10}$.

\section{- Mecanismos celulares}

A irradiação do laser de baixa potência pode levar a um efeito anti-inflamatório pela redução dos níveis de citocinas próinflamatórias $^{23}$ aumento na expressão de proteínas relacionadas à proliferação e maturação (colágeno), e inibição da produção de prostaglandina E2 (PGE2).

Vários tipos de células podem ter seus níveis de proliferação aumentados pelo LLLT. O laser pode induzir uma proliferação e maturação de células epiteliais, os queratinócitos ${ }^{40}$, e fibroblastos, demonstrando 0 efeito bioestimulador da terapia de fotobiomodulação e a utilidade dessa terapia no processo de cicatrização de feridas. Os efeitos bioestimulantes dessa terapia também atingem as células endoteliais, que são estimuladas à proliferação e angiogênese, e osteoblastos no tecido ósseo, aumentando a proliferação e diferenciação osteoblástica no processo de reparo ${ }^{41}$.

- A eficácia clínica do laser de baixa potência na resolução de lesões nervosas e disordens neurossensoriais

Dependendo da gravidade das lesões nervosas, tratamentos diferentes são propostos para a recuperação neurossensorial. Embora a microneurocirurgia possa ser eficaz para lesões nervosas graves (neurotmese), não existe um protocolo bem documentado para níveis mais baixos de lesões, ou seja, neuropraxia. A tecnologia a laser pode fornecer uma abordagem não invasiva para o tratamento de lesões nervosas moderadas e ser um método auxiliar em lesões nervosas graves ${ }^{42}$.

A terapia de fotobiomodulação (PBMT) vem sendo investigada devido à sua capacidade de estímulo aos processos de reparo tecidual, como em casos de danos aos nervos periféricos ${ }^{43}$. A luz utilizada geralmente possui largura espectral estreita entre $600 \mathrm{~nm}-1000$ $\mathrm{nm}$, podendo ser no espectro de luz vermelha ou infravermelha, e é frequentemente aplicada à lesão por 30 segundos a alguns minutos ou mais, algumas vezes por semana durante várias semanas, por todo o trajeto do nervo ${ }^{44}$.

Segundo uma revisão sistemática feita por Rosso et al. ${ }^{45}$, a fotobiomodulação possui efetividade na melhora dos aspectos morfológicos do nervo periférico regenerado, bem como na redução da resposta inflamatória e dolorosa, proporcionando recuperação funcional mais rápida e de melhor qualidade.

Em um estudo em que foi verificada a eficácia do uso de um protocolo de laserterapia de baixo nível, com laser infravermelho (780nm), após cirurgia ortognática, verificou-se melhoras de resposta do tecido e aceleração da recuperação de distúrbios neurossensoriais após a osteotomia bilateral sagital da mandíbula ${ }^{46}$.

Outros achados dos efeito benéficos desta terapia na restauração da função neurossensorial do nervo mandibular foram demonstrados por Führer-Valdivia et al. $^{47}$, em que a recuperação normal foi alcançada por um número maior de pacientes do grupo experimental, em detrimento do grupo controle, sugerindo um efeito benéfico da terapia a laser de baixo nível $(810+/-20 \mathrm{~nm}, 100 \mathrm{~mW})$ no comprometimento neurossensorial do nervo 
mandibular durante os primeiros 6 meses após a cirurgia ortognática.

Segundo Santo et al. ${ }^{48}$ a recuperação neurossensorial pode ser recuperada de forma mais rápida se 0 tratamento for iniciado precocemente (até 30 dias após a cirurgia), possuindo melhoras mais significativas do que 0 tratamento no pós-operatório tardio ( 6 meses a 1 ano), já que o LLLT não proporcionou recuperação mais rápida de anormalidades sensoriais na área avaliada nestes casos. Portanto, estabelece-se que o tratamento com laser de baixa intensidade (LLLT) mostrou-se eficaz na recuperação de distúrbios neurossensoriais após cirurgia ortognática, durante o curto período pós-operatório.

A aplicação desta terapia após exodontias de terceiros molares também se mostrou significativamente eficaz na redução da dor, trismo e inchaço pós-operatórios, segundo Landucci et al. ${ }^{49}$. Os resultados deste estudo demonstraram que uma única dose do laser infravermelho (comprimento de onda $780 \mathrm{~nm}$ ) é eficaz na redução do desconforto após a cirurgia de extração do terceiro molar porque a radiação penetra profundamente nos tecidos. Devido à melhor profundidade de penetração, lasers diodos infravermelhos são recomendados para locais de destino mais profundos como o nervo alveolar inferior, que fica dentro do canal mandibular.

Embora a terapia PBM com lasers de diodo de 810 e $980 \mathrm{~nm}$ promovam a recuperação neurossensorial de IAN após lesão por esmagamento em ratos, avaliado segundo pesquisa in vivo, os resultados das análises imunológicas mostraram que os ratos do grupo do laser de $810 \mathrm{~nm}$ apresentaram níveis mais baixos de TNF- $\alpha$ e IL-1 $\beta$ (citocinas) em comparação com os do grupo do laser de 980 $\mathrm{nm}$. Portanto, verificou-se que mais melhorias são alcançadas pela aplicação do laser de 810 $\mathrm{nm}$ na modulação de biomarcadores imunológicos em lesões nervosas ${ }^{50}$.

DISCUSSÃO

Atualmente, no Brasil, os aparelhos de laser de baixa intensidade tem se tornado cada vez mais popularizados no meio odontológico, dada a sua ampla área de utilização entre as diferentes especialidades, sendo cada vez mais comum os cirurgiões-dentistas passarem a adquirir esta tecnologia em seus consultórios. Porém, ao adquirir o aparelho de laser, é importante que o profissional conheça as características, os efeitos do laser no meio biológico e sua forma de aplicação.

Todos os conceitos histológicos e anatômicos dos nervos periféricos são muito importantes para entender os mecanismos nervosos frente a uma injúria, seus efeitos clínicos na sintomatologia do paciente, no diagnóstico e no tratamento da parestesia, haja vista que os diferentes graus de lesão nervosa podem levar a diferentes sintomas clínicos e diferentes tratamentos. Além disso, o profissional também deve dominar os tipos de lesões nervosas visando um melhor diagnóstico e indicação de tratamento.

Diante das mais variadas causas que podem levar aos distúrbios neurossensoriais, sabe-se que o planejamento cirúrgico préoperatório é de grande importância para o sucesso do procedimento a ser executado. Com - avanço da tecnologia radiográfica, muitos profissionais passaram a indicar CTCB para análise dos potenciais fatores de risco e grau de dificuldade do procedimento.

Segundo Ghaeminia et al. ${ }^{51}$, a imagem CTCB contribui para um planejamento cirúrgico mais abrangente e avaliação de riscos na cirurgia de terceiros molares, o que pode minimizar o risco de lesão nervosa e alterar a abordagem cirúrgica. Segundo Korkmaz et al. ${ }^{52}$, a tomografia computadorizada pode diminuir a prevalência de lesão temporária do $\mathrm{n}$. alveolar inferior e melhorar os resultados cirúrgicos em pacientes de alto risco de lesão nervosa.

No entanto, Clé-Ovejero et al. ${ }^{29}$ concluíram que apesar das imagens de tomografia pré-operatórias poderem ser úteis em termos de diagnóstico na extração de terceiros molares inferiores, ter essas imagens não reduz o risco dos pacientes às lesões ao NAI. Portanto, não deve realizar TC rotineiramente antes da cirurgia do $3 \mathrm{M}$ porque 0 uso de imagens tomográficas não parece reduzir a incidência de lesões ou afetar o prognóstico do paciente, em comparação com o uso de imagens panorâmicas.

Assim, de acordo com Kang et al. ${ }^{53}$, os principais fatores determinantes do risco de dano e distúrbio neurossensorial ao $\mathrm{n}$. alveolar inferior após extrações de terceiros molares são achados radiográficos, como estreitamento do canal mandibular $(19,8 \%)$ e escurecimento da raiz $(18,9 \%)$, grau de impacção óssea, proximidade do dente ao canal mandibular, a técnica cirúrgica de escolha (necessidade de ostectomia revelou maior risco), a exposição do nervo no período intraoperatório e experiência do cirurgião, sendo assim recomendado a TCCB quando estes sinais forem identificados na radiografia panorâmica.

$\mathrm{Na}$ odontologia clínica, a lesão iatrogênica do nervo lingual é uma complicação significativa e pode trazer grandes prejuízos à 
qualidade de vida do paciente. O NL pode ser danificado no espaço pterigomandibular e área do terceiro molar, durante procedimentos de rotina como bloqueios anestésicos e exodontia de terceiros molares, sendo a área retromolar um novo marco para evitar lesão iatrogênica de NL.54 Além disso, melhor treinamento na administração de bloqueios nervosos alveolares inferiores e o uso de técnicas anestésicas alternativas quando possíveis são recomendados para diminuir a ocorrência de trauma não intencional do nervo lingual.

Ainda hoje, existem poucas evidências para assegurar a eficácia clínica do laser de baixa potência na terapia de fotobiomodulação na resolução de lesões nervosas. Alguns ensaios clínicos randomizados concluem que a terapia a laser de baixa potência foi benéfica na recuperação do comprometimento neurossensorial do nervo mandibular, de acordo com Führer-Valdivia ${ }^{47}$, em comparação com um placebo.

Santo et al. ${ }^{48}$ também mostraram, em um estudo randomizado com vinte pacientes que, após osteotomia do ramo da mandíbula, tiveram parestesia, o lado de aplicação do LLLT exibiu uma melhora significativa na recuperação neurossensorial ao longo das sessões, além de que o tratamento precoce produziu melhores resultados.

Em contrapartida, alguns estudos não puderam fornecer evidências suficientes para concluir que existe melhoria neurossensorial para o grupo que recebeu LLLT como forma de tratamento, em relação ao que recebeu placebo, diante de lesões ao NAl ou NL.55 Existe, portanto, uma necessidade contínua de mais estudos clínicos sobre LLLT em lesões nervosas trigeminais.

Outro fator a ser levado em consideração, são as diferenças quanto ao comprimento de onda, parâmetros de irradiação e dosimetria utilizados nos estudos descritos na literatura que analisam a efetividade do LLLT em lesões nervosas, pois dificulta a obtenção de informações claras e objetivas que comprovariam e facilitariam a aplicação clínica do laser de baixa potência pelo profissional de saúde.3 Isso evidencia a necessidade de mais estudos clínicos para a elaboração de protocolos seguros e eficazes na aplicação do LLLT em lesões nervosas periféricas

CONCLUSÃO

O reparo das lesões nervosas póstraumáticas trigeminais representa um grande desafio para os cirurgiões-dentistas, no que se refere ao tratamento a ser indicado. O uso do laser de baixa potência na terapia da fotobiomodulação tem apresentado grandes benefícios, com fenômenos analgésicos, antiinflamatórios, cicatrizantes e regenerativos, acelerando o processo de reparo. Embora tenha havido grandes descobertas nos últimos anos, a literatura ainda carece de protocolos e ensaios clínicos duplo-cegos controlados verificando e comprovando esses efeitos sobre lesões nervosas, devendo este ser o foco de pesquisas futuras.

Além disso, o profissional de odontologia deve estar atento às principais causas e fatores de risco aos traumas nervosos que levam aos distúrbios neurossensoriais, para que possa haver adequado planejamento do procedimento e prevenção às injúrias nervosas. Em procedimento cirúrgicos, como extração de terceiros molares, colocação de implantes e cirurgias ortognáticas, as tomografias podem ser indicadas por haver risco aumentado de dano, embora não haja evidências de que o exame tomográfico diminua a incidência dos distúrbios neurossensoriais.

\section{REFERÊNCIAS}

1. Andrade, ED. Terapeutica Medicamentosa em Odontologia. 2014; 3eeição:107-109.

2. Kim, Y. K., Kim, S. G. \& Kim, J. H. Altered sensation after orthognathic surgery. J Oral Maxillofac Surg. 2011;69(3):893-98.

3. de Oliveira RF, de Andrade Salgado DM, Trevelin LT, Lopes RM, da Cunha SR, Aranha AC, de Paula Eduardo C, de Freitas PM. Benefits of laser phototherapy on nerve repair. Lasers Med Sci. 2015;30(4):1395-406.

4. Coulthard P, Kushnerev E, Yates JM, Walsh T, Patel N, Bailey E, Renton TF. Interventions for iatrogenic inferior alveolar and lingual nerve injury. Cochrane Database Syst Rev. 2014;16(4):CD00529

5. Al-Sabbagh M, Okeson JP, Bertoli E, Medynski DC, Khalaf MW. Persistent pain and neurosensory disturbance after dental implant surgery: prevention and treatment. Dent Clin North Am. 2015;59(1):143-56.

6. Anders JJ, Lanzafame RJ, Arany PR. Low-level light/laser therapy versus photobiomodulation therapy. Photomed Laser Surg. 2015;33(4):183-4.

7. Alves FRF, Dias MCC, Mansa MGCB, Machado MD. Permanent Labiomandibular Paresthesia after Bioceramic Sealer Extrusion: A Case Report. J Endod. 2020;46(2):301-6.

8. Akerzoul N, Chbicheb S. Low laser therapy as an effective treatment of recurrent aphtous ulcers: a clinical case reporting two locations. Pan Afr Med J. 2018;30:205.

9. Kalhori KAM, Vahdatinia F, Jamalpour MR, Vescovi P, Fornaini C, Merigo E, Fekrazad R. 
Photobiomodulation in Oral Medicine. Photobiomodul Photomed Laser Surg. 2019;37(12):837-61.

10. Hamblin MR. Mechanisms and applications of the anti-inflammatory effects of photobiomodulation. AIMS Biophys. 2017;4(3):337-61.

11. Piccinni C, Gissi DB, Gabusi A, Montebugnoli L, Poluzzi E. Paraesthesia after local anaesthetics: an analysis of reports to the FDA Adverse Event Reporting System. Basic Clin Pharmacol Toxicol. 2015;117(1):52-6.

12. Eshghpour M, Ahrari F, Takallu M. Is Low-Level Laser Therapy Effective in the Management of Pain and Swelling After Mandibular Third Molar Surgery? J Oral Maxillofac Surg. 2016; 74(7):1322.e1-8.

13. Khojastepour L, Khaghaninejad MS, Hasanshahi R, Forghani M, Ahrari F. Does the Winter or Pell and Gregory Classification System Indicate the Apical Position of Impacted Mandibular Third Molars? J Oral Maxillofac Surg. 2019;77(11):2222.e1-2222.e9

14.Zaroni FM, Cavalcante RC, João da Costa D, Kluppel LE, Scariot R, Rebellato NLB. Complications associated with orthognathic surgery: A retrospective study of 485 cases. J Craniomaxillofac Surg. 2019;47(12):18551860.

15. Roychoudhury S, Nagori SA, Roychoudhury A. Neurosensory disturbance after bilateral sagittal split osteotomy: A retrospective study. J Oral Biol Craniofac Res. 2015;5(2):65-8.

16. Perotti S, Bin P, Cecchi R. Hypochlorite accident during wndodontic therapy with nerve damage - A case report. Acta Biomed. 2018;89(1):104-108.

17. Alves FRF, Dias MCC, Mansa MGCB, Machado MD. Permanent Labiomandibular Paresthesia after Bioceramic Sealer Extrusion: A Case Report. J Endod. 2020;46(2):301-6.

18. Mahmood H, Stern M, Atkins S. Inferior alveolar nerve anaesthesia: $A$ rare complication of orthodontic tooth movement. J Orthod. 2019;46(4):374-377.

19. Menorca RM, Fussell TS, Elfar JC. Nerve physiology: mechanisms of injury and recovery. Hand Clin. 2013;29(3):317-30.

20. Tan VL, Andrawos A, Ghabriel MN, Townsend GC. Applied anatomy of the lingual nerve: relevance to dental anaesthesia. Arch Oral Biol. 2014;59(3):324-35.

21. Seddon HJ. Three types of nerve injury. in BRAIN. 1943;66: 238-83.

22. Sunderland S. A classification of peripheral nerve injuries producing loss of function. Brain. 1951;74(4):491-516.

23. de Freitas LF, Hamblin MR. Proposed Mechanisms of Photobiomodulation or Low-
Level Light Therapy. IEEE J Sel Top Quantum Electron. 2016;22(3):7000417.

24. Sánchez-Torres A, Soler-Capdevila J, UstrellBarral M, Gay-Escoda C. Patient, radiological, and operative factors associated with surgical difficulty in the extraction of third molars: a systematic review. Int J Oral Maxillofac Surg. 2020;49(5):655-65.

25. Huang CK, Lui MT, Cheng DH. Use of panoramic radiography to predict postsurgical sensory impairment following extraction of impacted mandibular third molars. J Chin Med Assoc. 2015;78(10):617-22.

26. Su N, van Wijk A, Berkhout E, Sanderink G, De Lange J, Wang $\mathrm{H}$, van der Heijden GJMG. Predictive Value of Panoramic Radiography for Injury of Inferior Alveolar Nerve After Mandibular Third Molar Surgery. J Oral Maxillofac Surg. 2017;75(4):663-79.

27. Araujo GTT, Peralta-Mamani M, Silva AFMD, Rubira CMF, Honório HM, Rubira-Bullen IRF. Influence of cone beam computed tomography versus panoramic radiography on the surgical technique of third molar removal: a systematic review. Int $\mathrm{J}$ Oral Maxillofac Surg. 2019;48(10):1340-347.

28. de Toledo Telles-Araújo G, Peralta-Mamani M, Caminha RDG, de Fatima Moraes-da-Silva A, Rubira CMF, Honório HM, Rubira-Bullen IRF. CBCT does not reduce neurosensory disturbances after third molar removal compared to panoramic radiography: a systematic review and meta-analysis. Clin Oral Investig. 2020;24(3):1137-149.

29. Clé-Ovejero A, Sánchez-Torres A, Camps-Font O, Gay-Escoda C, Figueiredo R, ValmasedaCastellón E. Does 3-dimensional imaging of the third molar reduce the risk of experiencing inferior alveolar nerve injury owing to extraction?: A meta-analysis. J Am Dent Assoc. 2017;148(8):575-83

30. Lin CS, Wu SY, Huang HY, Lai YL. Systematic Review and Meta-Analysis on Incidence of Altered Sensation of Mandibular Implant Surgery. PLoS One. 2016;11(4):e0154082.

31. Başa O, Dilek OC. Assessment of the risk of perforation of the mandibular canal by implant drill using density and thickness parameters. Gerodontology. 2011;28(3):213-20.

32. Scarano A, Sinjari B, Murmura G, Lorusso F. Neurosensory Disturbance of the Inferior Alveolar Nerve After 3025 Implant Placements. Implant Dent. 2017;26(5):735-43.

33. Bittencourt MA, Paranhos LR, Martins-Filho PR. Low-level laser therapy for treatment of neurosensory disorders after orthognathic surgery: A systematic review of randomized clinical trials. Med Oral Patol Oral Cir Bucal. 2017;22(6):780-87. 
34. McLeod NM, Bowe DC. Nerve injury associated with orthognathic surgery. Part 2: inferior alveolar nerve. $\mathrm{Br}$ J Oral Maxillofac Surg. 2016 May;54(4):366-71.

35. Demirbas AE, Yilmaz G, Topan C, Kütük N, Kilic E, Alkan A. Risk Factors Influencing Recovery of Neurosensory Disturbances Following Sagittal Split Ramus Osteotomy. J Craniofac Surg. 2020;31(1):e35-8.

36. Pippi R, Spota A, Santoro M. Medicolegal Considerations Involving latrogenic Lingual Nerve Damage. J Oral Maxillofac Surg. 2018;76(8):1651.e1-1651.e13.

37. Luke AM, Mathew S, Altawash MM, Madan BM. Lasers: A Review With Their Applications in Oral Medicine. J Lasers Med Sci. 2019;10(4):324-29.

38. Farivar S, Malekshahabi T, Shiari R. Biological effects of low level laser therapy. J Lasers Med Sci. 2014;5(2):58-62

39. Pannala VR, Camara AK, Dash RK. Modeling the detailed kinetics of mitochondrial cytochrome c oxidase: Catalytic mechanism and nitric oxide inhibition. J Appl Physiol (1985). 2016;121(5):1196-207.

40. Esmaeelinejad M, Bayat M, Darbandi H, Bayat M, Mosaffa N. The effects of low-level laser irradiation on cellular viability and proliferation of human skin fibroblasts cultured in high glucose mediums. Lasers Med Sci. 2014;29(1):121-29.

41. Effect of low level laser therapy on proliferation and differentiation of the cells contributing in bone regeneration. $J$ Lasers Med Sci. 2014;5(4):163-70

42. Hakimiha $N$, Dehghan MM, Manaheji $H$, Zaringhalam J, Farzad-Mohajeri S, Fekrazad R, Moslemi N. Recovery of inferior alveolar nerve by photobiomodulation therapy using two laser wavelengths: A behavioral and immunological study in rat. $\mathrm{J}$ Photochem Photobiol B. 2020;204:111785.

43. Huang YY, Sharma SK, Carroll J, Hamblin MR. Biphasic dose response in low level light therapy - an update. Dose Response. 2011;9(4):602-18.

44. Garcez AS, Ribeiro MS, Núnez SC. LASER de baixa potência princípios básicos e aplicações clínicas na odontologia. Rio de Janeiro: Ed Elsevier; 2012.

45. Rosso MPO, Buchaim DV, Kawano N, Furlanette G, Pomini KT, Buchaim RL. Photobiomodulation Therapy (PBMT) in Peripheral Nerve Regeneration: A Systematic Review. Bioengineering (Basel). 2018;5(2):44.

46. Gasperini G, de Siqueira IC, Costa LR. Lowerlevel laser therapy improves neurosensory disorders resulting from bilateral mandibular sagittal split osteotomy: a randomized crossover clinical trial. J Craniomaxillofac Surg. 2014;42(5):e130-33.

47. Führer-Valdivia $A, \quad$ Noguera-Pantoja $A$, Ramírez-Lobos V, Solé-Ventura P. Low-level laser effect in patients with neurosensory impairment of mandibular nerve after sagittal split ramus osteotomy. Randomized clinical trial, controlled by placebo. Med Oral Patol Oral Cir Bucal. 2014;19(4):e327-34.

48. Santos FT, Sciescia R, Santos PL, Weckwerth V, Dela Coleta Pizzol KE, Queiroz TP. Is LowLevel Laser Therapy Effective on Sensorineural Recovery After Bilateral Sagittal Split Osteotomy? Randomized Trial. J Oral Maxillofac Surg. 2019;77(1):164-73.

49. Landucci A, Wosny AC, Uetanabaro LC, Moro A, Araujo MR. Efficacy of a single dose of lowlevel laser therapy in reducing pain, swelling, and trismus following third molar extraction surgery. Int $\mathrm{J}$ Oral Maxillofac Surg. 2016;45(3):392-98.

50. Hakimiha $N$, Dehghan MM, Manaheji $H$, Zaringhalam J, Farzad-Mohajeri S, Fekrazad R, Moslemi N. Recovery of inferior alveolar nerve by photobiomodulation therapy using two laser wavelengths: A behavioral and immunological study in rat. $\mathrm{J}$ Photochem Photobiol B. 2020;204:111785.

51. Ghaeminia H, Meijer GJ, Soehardi A, Borstlap WA, Mulder J, Vlijmen OJ, Bergé SJ, Maal TJ. The use of cone beam CT for the removal of wisdom teeth changes the surgical approach compared with panoramic radiography: a pilot study. Int $\mathrm{J}$ Oral Maxillofac Surg. 2011;40(8):834-39.

52. Korkmaz YT, Kayıpmaz S, Senel FC, Atasoy KT, Gumrukcu Z. Does additional cone beam computed tomography decrease the risk of inferior alveolar nerve injury in high-risk cases undergoing third molar surgery?Does CBCT decrease the risk of IAN injury? Int J Oral Maxillofac Surg. 2017;46(5):628-35.

53. Kang F, Sah MK, Fei G. Determining the risk relationship associated with inferior alveolar nerve injury following removal of mandibular third molar teeth: A systematic review. J Stomatol Oral Maxillofac Surg. 2020;121(1): 63-69.

54. Iwanaga J, Cleveland MK, Wada J, Tubbs RS. How to avoid iatrogenic lingual nerve injury in the retromolar area: an anatomical study of retromolar pad and lingual nerve. Surg Radiol Anat. 2020;42(5):523-28.

55. Miloro M, Criddle TR. Does Low-Level Laser Therapy Affect Recovery of Lingual and Inferior Alveolar Nerve Injuries? J Oral Maxillofac Surg. 2018;76(12):2669-675. 


\section{CONFLITO DE INTERESSES}

Os autores declaram não haver conflitos de interesse

\section{AUTOR PARA CORRESPONDÊNCIA}

\section{Ellen Cristina Gaetti Jardim}

Faculdade de Odontologia (FAODO)

Universidade Federal de Mato Grosso do Sul (UFMS)

79070-900 Campo Grande - MS, Brasil

E-mail: ellen.jardim@ufms.br

Submetido em 09/12/2020

Aceito em 16/07/2021 